\title{
Órgãos vitais e trato gastrintestinal de vacas de descarte mestiças Charolês $\times$ Nelore abatidas com pesos distintos ${ }^{1}$
}

\author{
Fernando Kuss ${ }^{2}$, João Restle ${ }^{3}$, Ivan Luiz Brondani ${ }^{4}$, Paulo Santana Pacheco ${ }^{5}$, Magali \\ Floriano da Silveira ${ }^{6}$, Raul Dirceu Pazdiora ${ }^{7}$, Ian Machado Cezimbra ${ }^{8}$
}

\author{
1 Parte da dissertação apresentada à UFSM pelo primeiro autor para obtenção do título de Mestre em Produção Animal. \\ 2 Doutorando do Programa de Pós-Graduação em Zootecnia da UFRGS. Bolsista CNPq. \\ 3 Professor visitante do CNPq - Departamento de Produção Animal - EV/UFG, Campus Samambaia, CP 131 - Goiânia - GO. \\ 4 Departamento de Zootecnia da UFSM. \\ 5 Doutorando do PPGCA-UFG. \\ 6 PPG em Zootecnia - UFSM. \\ 7 Graduando em Medicina Veterinária da UFSM. \\ 8 Graduando em Zootecnia da UFSM.
}

RESUMO - Objetivou-se estudar o desenvolvimento dos órgãos vitais e do trato gastrintestinal de vacas de descarte mestiças provenientes da segunda (G2 - 3/4Charolês (C) 1/4Nelore (N) e 3/4NC) e terceira geração (G3 - 5/8CN e 5/8NC) do cruzamento rotativo Charolês $\times$ Nelore terminadas em confinamento e abatidas com 465 (T465), 507 (T507) ou 566 kg (T566). Os animais apresentaram, em média, ao início do confinamento, 8,5 anos, 388,6 kg e 2,35 pontos de escore corporal. A dieta, com relação volumoso:concentrado de 48:52, continha 12,5\% de PB e 2,99 Mcal de ED/kg de MS. Entre os órgãos, o fígado apresentou maior desenvolvimento. O pulmão e o baço desenvolveram até os animais atingirem $507 \mathrm{~kg}$, reduzindo aos $566 \mathrm{~kg}$. O aumento do peso de abate conferiu menor participação relativa do conjunto dos órgãos vitais e trato gastrintestinal no peso vivo e no corpo vazio. Animais da terceira geração do cruzamento rotativo Charolês $\times$ Nelore apresentaram maior peso de rúmen-retículo e estômago (14,29 vs $12,30 \mathrm{~kg}$ e 24,43 vs 22,28 kg, respectivamente) em relação àqueles da segunda geração. Entretanto, quando o peso dos órgãos foi corrigido para peso vivo e peso de corpo vazio, somente o rúmen-retículo foi maior na terceira geração. Maior participação de sangue Charolês no genótipo conferiu maior volume de sangue, desenvolvimento dos pulmões e do conjunto dos órgãos vitais em relação ao corpo vazio.

Palavras-chave: bovinos de corte, cruzamento, estômago, fêmeas, fígado

\section{Vital organs and gastrointestinal tract of feedlot finished Charolais $\mathrm{x}$ Nellore culling crossbred cows slaughtered at different weights}

\begin{abstract}
The objective of this experiment was to study the development of vital organs and gastrointestinal tract of culling crossbred cows from the second (G2: 3/4Charolais (C) Nellore (N) and 3/4NC) and third (G3: 5/8CN and 5/8NC) generations of C x N rotational crossbreeding. Animals were feedlot finished and slaughtered with 465 (T465), 507 (T507), and $566 \mathrm{~kg}$ (T566) of body weight (BW). At the beginning of the trial, age, BW, and body condition averaged respectively, 8.5 years, $388.6 \mathrm{~kg}$ and, 2.35 points. Animals were fed a 48:52 forage:concentrate ratio diet containing $12.5 \%$ crude protein and $2.99 \mathrm{Mcal}$ of digestible energy per kg of dry matter. Liver showed the greatest development among the studied organs. Lungs and spleen showed high development until animals reached $507 \mathrm{~kg} \mathrm{BW}$ (T507) but reduced at $566 \mathrm{~kg}$ BW (T566). Increasing slaughter weight reduced the proportion of internal organs and gastrointestinal tract in the total BW and empty body weight (EBW). G3 animals had heavier reticulum-rumen and stomach (reticulum-rumen+omasum+abomasum) (14.29 vs. $12.30 \mathrm{~kg}$ and 24.43 vs. $22.28 \mathrm{~kg}$, respectively) compared to G2 cows. However, when expressed as proportion of BW and EBW only the reticulum-rumen weight differed between G2 and G3 cows. The greater contribution of C in the genotype resulted in increased volume of blood and development of lungs and all vital organs together when expressed as proportions of EBW.
\end{abstract}

Key Words: beef cattle, cows, crossbreeding, liver, stomach

\section{Introdução}

É evidente a preocupação dos frigoríficos em quantificar os componentes não pertencentes à carcaça no intuito de maximizar o ganho econômico dos animais abatidos. Entre os componentes de maior valorização, destacam-se couro, coração, fígado, rins e intestinos. Os órgãos vitais (coração, fígado, rins e intestinos), no entanto, podem representar de 2 a $4 \%$ do valor comercial da carcaça, de modo que o tamanho destes tecidos pode influenciar o rendimento da 
carcaça. No estudo de Vaz et al. (2001), o menor rendimento de carcaça das novilhas em relação aos novilhos superprecoces Braford foi justificado pelo maior percentual do peso vivo dos órgãos internos das fêmeas.

As diferenças no tamanho dos órgãos internos, entre raças, podem estar associadas às exigências de mantença. No NRC (1996), as diferenças entre espécies de bovídeos variam de acordo com as exigências de mantença, o peso vivo, a categoria animal e o desempenho esperado. Segundo Caton \& Dhuyvetter (1997), em ruminantes adultos, a maior parte da energia utilizada para mantença é consumida pelas vísceras. Smith \& Baldwin (1973) mencionam que o coração, o fígado e o trato gastrintestinal estão entre os tecidos de maior atividade metabólica nos animais. Oliveira et al. (1992) observaram em animais mais leves maior desenvolvimento do trato gastrintestinal, como resultado do fato de as vísceras atingirem desenvolvimento máximo em tempo menor que o tecido ósseo e muscular.

Resultados de pesquisas comprovaram haver alterações no peso do trato gastrintestinal e dos órgãos internos de animais zebuínos em comparação a taurinos e mestiços (Lunt et al., 1986; Solis et al., 1988; Fernandes et al., 2002), demonstrando menor exigência de mantença para os animais zebuínos, como reflexo da menor atividade metabólica dos órgãos e da quantidade de gordura interna.

Além do fator genético, pesquisas evidenciam influência do estádio de maturidade sobre os órgãos internos de ruminantes. Jorge et al. (1999) observaram aumento linear do peso dos órgãos vitais e dos intestinos em relação ao peso corporal vazio com o aumento do peso de abate de novilhos abatidos em diferentes estádios de maturidade. Esses autores reportaram ainda que, entre estes componentes, o fígado e o baço são os órgãos de maior ímpeto de crescimento (Jorge \& Fontes, 2001). Restle et al. (2005) demonstraram que o aumento do peso de abate de novilhos 5/8 Nelore 3/8 Charolês terminados em confinamento pro porcionou redução linear do conjunto total dos órgãos vitais em relação ao corpo vazio e observaram similar desenvolvimento do trato gastrintestinal entre os pesos de abate preconizados (425, 467 e $510 \mathrm{~kg}$ de PV).

Do total do rebanho bovino destinado ao abate no Brasil, matriz ou vaca de cria foi a categoria animal que apresentou aumento significativo na última década $(16,4 \%)$, enquanto as demais mantiveram-se estáveis ou até diminuíram em número de animais. A taxa de abate de fêmeas no Brasil e no Rio Grande do Sul representou, no ano de $2003,46,1$ e $51,2 \%$ do abate total de bovinos, respectivamente (ANUALPEC, 2004).

Este trabalho foi realizado com objetivo de avaliar o desenvolvimento biométrico dos órgãos vitais e do trato gastrintestinal de vacas de descarte mestiças Charolês $\times$ Nelore abatidas com diferentes pesos.

\section{Material e Métodos}

O estudo foi conduzido no Setor de Bovinocultura de Corte do Departamento de Zootecnia da Universidade Federal de Santa Maria, em Santa Maria-RS.

Foram utilizadas 24 vacas de descarte resultantes do cruzamento alternado Charolês $(\mathrm{C}) \times$ Nelore $(\mathrm{N})$, sendo 12 animais da segunda ( $\mathrm{G} 2$ - seis vacas $3 / 4 \mathrm{C}-1 / 4 \mathrm{~N}$ e seis vacas $3 / 4 \mathrm{~N}-1 / 4 \mathrm{C})$ e 12 da terceira geração (G3 - seis vacas $5 / 8 \mathrm{C}$ $3 / 8 \mathrm{~N}$ e seis vacas $5 / 8 \mathrm{~N}-3 / 8 \mathrm{C}$ ). Ao início do período experimental, os animais apresentavam, em média, 8,5 anos de idade, 388,6 kg de PV e 2,35 pontos de escore da condição corporal, segundo a classificação proposta por Restle (1972), em que $1=$ muito magro, $2=$ magro, $3=$ médio, $4=$ gordo $\mathrm{e}$ $5=$ muito gordo.

Os tratamentos consistiram de três faixas de peso de abate. Os pesos de abate inicialmente pretendidos eram de 460,510 e $560 \mathrm{~kg}$, mas os pesos reais foram 465, 507 e $566 \mathrm{~kg}$. Para atingir os pesos determinados, os animais foram mantidos em confinamento e alimentados com uma dieta contendo $12,5 \%$ de $\mathrm{PB}$ e 2,99 Mcal de ED/kg de MS, composta de $48 \%$ de volumoso (silagem de milho - AG 5011) e $52 \%$ de concentrado contendo $92,6 \%$ de farelo de trigo, $6,8 \%$ de calcário calcítico e $0,6 \%$ sal comum, com base na MS. Os animais foram alimentados duas vezes ao dia, às 9 e $16 \mathrm{~h}$, mantendo-se uma oferta diária de MS 10\% superior ao consumo voluntário.

À medida que a média dos lotes dos tratamentos atingiram o peso de abate pré-determinado, os animais foram submetidos a jejum de sólidos (12 horas), sendo pesados e transportados em caminhão boiadeiro por $25 \mathrm{~km}$ até o frigorífico comercial, onde foram abatidos logo após o desembarque, conforme o fluxo de abate normal do estabelecimento. Foi considerado peso de abate o peso final tomado na fazenda.

Foram separados da carcaça e pesados os componentes externos (sangue; cabeça com orelhas e chifres; pés; vassoura da cauda; couro e úbere), os órgãos vitais (coração, rins, fígado, baço e pulmões), o trato gastrintestinal (rúmenretículo, abomaso e omaso (estômago cheio e vazio), os intestinos delgado e grosso (cheio e vazio), a gordura total (gorduras visceral, de toalete e renal) e o aparelho reprodutivo.

Para obtenção do peso corporal vazio, utilizou-se o somatório dos pesos de carcaça quente, sangue, componentes externos, órgãos vitais, trato gastrintestinal vazio, aparelho reprodutivo e gordura total. 
$\mathrm{O}$ delineamento experimental utilizado foi o inteiramentecasualizado com arranjo fatorial 3 x 4 . Os dados foram submetidos à análise de normalidade pelo teste ShaphiroWilk e à análise de variância pelo teste $\mathrm{F}$.

O modelo estatístico empregado foi o seguinte:

$$
Y_{i j k l}=\mu+P_{i}+G_{j}+G_{K}\left(G_{J}\right)+P_{l}+E_{i j k l} \text {, }
$$

em que: $Y_{i j k l}=$ variáveis dependentes; $\mu=$ média de todas as observações; $\mathrm{Pa}_{\mathrm{i}}=$ efeito do peso de abate de ordem $\mathrm{i}$; $\mathrm{G}_{\mathrm{j}}=$ efeito da geração de cruzamento de ordem $\mathrm{j} ; \mathrm{GG}_{\mathrm{K}}\left(\mathrm{G}_{\mathrm{J}}\right)=$ efeito do grupo genético de ordem $\mathrm{k}$, aninhado dentro da geração de cruzamento de ordem $\mathrm{j} ; \mathrm{PI}_{1}=$ efeito da covariável peso inicial de ordem $1 ; \mathrm{E}_{\mathrm{ijkl}}=$ efeito aleatório residual.

As interações peso de abate $\times$ geração de cruzamento e grupo genético $\times$ geração foram inicialmente testadas e removidas do modelo estatístico final, pois não foram significativas para as variáveis avaliadas. As médias das variáveis dependentes significativamente afetadas pelas variáveis independentes, segundo análise de variância, foram comparadas pelo teste T (SAS, 1997).

Realizou-se, ainda, a análise de contraste entre animais de predominância Charolês $(\mathrm{PC}=3 / 4 \mathrm{CN}+5 / 8 \mathrm{CN})$ e Nelore $(\mathrm{PN}=3 / 4 \mathrm{NC}+5 / 8 \mathrm{CN})$.

\section{Resultados e Discussão}

Não houve efeito das interações $(\mathrm{P}>0,05)$ peso de abate $\times$ geração de cruzamento ou peso de abate $\times$ grupo genético da vaca sobre as variáveis estudadas e, por isso, as variáveis serão apresentadas e discutidas separadamente.

Na Tabela 1 são descritos os valores médios para corpo vazio (CV), sangue e órgãos vitais, em valor absoluto, e a participação relativa $(\%)$ no peso de abate (PA) e no peso de corpo vazio (PCV), de acordo com os pesos de abate preconizados. Em valores absolutos, o volume de sangue não aumentou com o incremento do peso de abate, o que resultou em menor participação deste fluido no PA e PCV quando o peso de abate aumentou de 507 para $566 \mathrm{~kg}$. Comportamento contrário foi registrado por Restle et al. (2005), que observaram incremento linear do volume absoluto de sangue em novilhos abatidos com 425, 467 e $510 \mathrm{~kg}$ de PV, ao passo que, quando ajustados para PA e PCV, os valores entre os distintos pesos de abate foram similares.

São poucos os trabalhos nos quais foi avaliado o peso do sangue. Segundo alguns autores (Ribeiro et al., 2001; Pacheco et al., 2005), o aumento do volume de sangue está associado ao incremento dos órgãos vitais e do trato gastrintestinal, exigindo maior volume de sangue para manter a taxa metabólica dos animais. Neste estudo, os valores
Tabela 1 - Pesos de corpo vazio, coração, fígado, pulmões, rins, baço, conjunto dos órgãos vitais (coração + fígado + pulmões + rins + baço) (COV) de vacas de descarte abatidas com diferentes pesos

Table 1 - Means and standard errors for weights of empty body, heart, liver, lungs, kidneys, spleen, and vital organs set (heart + liver + lungs + kidneys + spleen) (COV) according to different slaughter weights

\begin{tabular}{|c|c|c|c|}
\hline \multirow[t]{2}{*}{$\begin{array}{l}\text { Característica } \\
\text { Trait }\end{array}$} & \multicolumn{3}{|c|}{$\begin{array}{l}\text { Peso de abate } \\
\text { Slaughter weight }\end{array}$} \\
\hline & T465 & Т507 & Т566 \\
\hline & \multicolumn{3}{|c|}{ Peso absoluto, $\mathrm{kg}$ (Fresh weight, $\mathrm{kg}$ ) } \\
\hline $\begin{array}{l}\text { Corpo vazio** } \\
\text { Empty body }\end{array}$ & $376,34 \pm 11,17 \mathrm{c}$ & \multicolumn{2}{|l|}{$412 \pm 11,20 \mathrm{~b} 4 \mathrm{c}$} \\
\hline Sangue (Blood) & $13,78 \pm 0,66$ & $14,78 \pm 0,66$ & $14,50 \pm 0,66$ \\
\hline Coração (Heart) & $1,66 \pm 0,10$ & $1,73 \pm 0,10$ & $1,76 \pm 0,10$ \\
\hline Fígado** (Liver) & $5,55 \pm 0,24 b$ & $6,28 \pm 0,24 a$ & $6,39 \pm 024 a$ \\
\hline Pulmão* (Lungs) & $4,72 \pm 0,26 b$ & $5,97 \pm 0,26 a$ & $5,27 \pm 0,26 b$ \\
\hline Rins (Kidneys) & $0,83 \pm 0,10$ & $0,88 \pm 0,10$ & $0,83 \pm 0,10$ \\
\hline Baço** (Spleen) & $1,04 \pm 0,11 \mathrm{~b}$ & $1,46 \pm 0,11 \mathrm{a}$ & $0,96 \pm 0,11 \mathrm{~b}$ \\
\hline \multirow[t]{2}{*}{$\mathrm{COV}^{* *}$} & $13,76 \pm 0,46 \mathrm{c}$ & $16,33 \pm 0,46 \mathrm{ab}$ & $15,21 \pm 0,46 b$ \\
\hline & \multicolumn{3}{|c|}{$\%$ do peso de abate (\% of slaughter weight) } \\
\hline Sangue**(Blood) & $2,97 \pm 0,12 \mathrm{a}$ & $2,93 \pm 0,12 \mathrm{a}$ & $2,57 \pm 0,12 b$ \\
\hline Coração (Heart) & $0,35 \pm 0,02$ & $0,34 \pm 0,02$ & $0,31 \pm 0,02$ \\
\hline Fígado (Liver) & $1,19 \pm 0,04$ & $1,23 \pm 0,04$ & $1,13 \pm 0,04$ \\
\hline Pulmão** (Lungs) & $1,02 \pm 0,05 b$ & $1,17 \pm 0,05 \mathrm{a}$ & $0,93 \pm 0,05 b$ \\
\hline Rins (Kidneys) & $0,18 \pm 0,02$ & $0,18 \pm 0,02$ & $0,15 \pm 0,02$ \\
\hline Baço**(Spleen) & $0,22 \pm 0,02 b$ & $0,29 \pm 0,02 \mathrm{a}$ & $0,17 \pm 0,02 b$ \\
\hline \multirow[t]{2}{*}{$\mathrm{COV}^{* *}$} & $2,96 \pm 0,07 b$ & $3,21 \pm 0,07 \mathrm{a}$ & $2,69 \pm 0,07 \mathrm{c}$ \\
\hline & \multicolumn{3}{|c|}{$\%$ do corpo vazio ( $\%$ of empty body) } \\
\hline Sangue (Blood) & $3,66 \pm 0,14 \mathrm{a}$ & $3,61 \pm 0,14 \mathrm{a}$ & $3,14 \pm 0,14 b$ \\
\hline Coração (Heart) & $0,44 \pm 0,02$ & $0,42 \pm 0,02$ & $0,38 \pm 0,02$ \\
\hline Fígado* (Liver) & $1,46 \pm 0,05 \mathrm{ab}$ & $1,51 \pm 0,05 \mathrm{a}$ & $1,38 \pm 0,05 b$ \\
\hline Pulmão**(Lungs) & $1,26 \pm 0,06 b$ & $1,45 \pm 0,06 \mathrm{a}$ & $1,14 \pm 0,06 b$ \\
\hline Rins (Kidneys) & $0,22 \pm 0,02$ & $0,22 \pm 0,02$ & $0,18 \pm 0,02$ \\
\hline Baço* (Spleen) & $0,27 \pm 0,03 b$ & $0,36 \pm 0,03 \mathrm{a}$ & $0,20 \pm 0,03 \mathrm{c}$ \\
\hline $\mathrm{COV}^{* *}$ & $3,65 \pm 0,08 b$ & $3,95 \pm 0,08 \mathrm{a}$ & $3,28 \pm 0,08 \mathrm{c}$ \\
\hline
\end{tabular}

$a, b, c$ : médias na mesma linha diferem ** $(P<0,05)$ ou * $(P<0,10)$ pelo teste $t$ $a, b, c$ : means in the same row differ ${ }^{* *}(P<0.05)$ or ${ }^{*}(P<0.10)$ by $t$ test.

absolutos de sangue e do conjunto de órgãos vitais (COV) apresentaram correlação significativa de 0,49 (Tabela 3).

Não houve efeito do peso de abate sobre o desenvolvimento de coração e rins, ambos expressos em valor absoluto e percentual do PA e PCV, sendo obtidos valores médios de $1,72 \mathrm{~kg}, 0,33 \%$ do PA e $0,41 \%$ do PCV para coração e de $0,85 \mathrm{~kg}, 0,17 \%$ do PA e $0,21 \%$ do PCV para rins. Estes resultados comprovam que a integridade desses órgãos não é afetada pelo peso de abate, não havendo aumento de demanda de nutrientes. Em novilhos com 22 meses de idade terminados em confinamento (abatidos com $450 \mathrm{~kg}$ de peso vivo) e alimentados com nível de concentrado similar ao utilizado neste experimento (50\% na MS), Véras et al. (2001) encontraram valores médios de 1,51 e $0,78 \mathrm{~kg}$ e 0,38 e $0,20 \%$ do PCV para coração e rins, respectivamente. Tamanho superior de coração em vacas com relação aos novilhos 
é esperado, haja vista a maior superfície corporal desses animais e a tendência de maior quantidade de tecido muscular neste órgão para suprir a demanda sangüínea nos demais tecidos corporais. Verificou-se associação positiva e significativa $(0,63)$ entre peso do coração e peso de abate (Tabela 3).

O peso de fígado aumentou do T465 para o T507 e manteve-se constante no peso de $566 \mathrm{~kg}$. Quando expresso em \% do CV, apresentou redução do T507 para o T566, indicando que o ganho compensatório foi mais intenso até o T507. Maior velocidade de deposição de massa corporal pode exigir do fígado maior demanda de síntese de nutrientes para formação de tecidos. A correlação entre o desenvolvimento do fígado e o ganho de peso dos animais ao longo do confinamento foi de 0,57 (Tabela 3).

Segundo Hoog (1991), durante o crescimento compensatório, grande parte das alterações observadas no peso do animal é resultante da recuperação da atividade metabólica do fígado e do intestino delgado e, conseqüentemente, do incremento do peso destes órgãos associado ao aumento do conteúdo da digesta. Essa tendência de aumento da digesta (Kuss et al., 2007) e do desenvolvimento dos intestinos foi observada também neste estudo (Tabela 2). Trabalhando com animais em fase de crescimento (20 a 24 meses de idade), Jorge \& Fontes (2001) e Restle et al. (2005) demonstraram elevado ímpeto de crescimento do fígado com a maturidade dos animais, acompanhando simultaneamente o crescimento do peso corporal vazio dos novilhos. Além do aumento do peso de abate, os resultados obtidos por Véras et al. (2001) demonstraram que o aumento do nível do concentrado na dieta também influencia o desenvolvimento do fígado.

Verificou-se desenvolvimento de pulmões e baço até o T507, com redução no T566 em todas as formas de expressão. Esta redução pode estar associada à maior pressão interna exercida sobre estes órgãos, principalmente pela gordura interna, e ao maior volume do trato gastrintestinal causado pelo aumento da digesta, sendo mais nítida nos animais do T566 (Kuss et al., 2007) (Tabela 2). Outra suposição seria o efeito do ganho de peso: ao longo do processo de terminação, os animais apresentaram ganho de peso médio diário de 1,26; 1,59 e 1,28 kg (T465, T507 e T566, respectivamente), ou seja, a maior velocidade de ganho de peso no T507 exigiu maior demanda de oxigenação (pulmões) e capacidade de armazenamento de sangue (baço), a fim de acompanhar a produção dos tecidos muscular e adiposo. Oganho de peso esteve positivamente correlacionado (correlações de 0,41 e 0,55 , respectivamente) ao peso desses órgãos (Tabela 3). Lunt et al. (1986) também associaram o ganho de peso de novilhos Angus, Brahman e Brahman x
Tabela 2 - Peso de rúmen-retículo, abomaso, omaso, estomago (rúmen-retículo + abomaso + omaso), intestinos (delgado + grosso) e trato gastrintestinal (estômago + intestinos) (TGI) de vacas de descarte abatidas com diferentes pesos

Table 2 - Means and standard errors for weights of reticulum-rumen, abomasum, omasum, stomach (reticulum-rumen + abomasum + omasum), intestines (small + large), gastrointestinal tract (stomach+intestines) (GIT) according to different slaughter weights

\begin{tabular}{|c|c|c|c|}
\hline \multirow[t]{2}{*}{$\begin{array}{l}\text { Característica } \\
\text { Item }\end{array}$} & \multicolumn{3}{|c|}{$\begin{array}{l}\text { Peso de abate } \\
\text { Slaughter weight }\end{array}$} \\
\hline & $\mathrm{T} 465$ & T507 & T566 \\
\hline & \multicolumn{3}{|c|}{ Peso absoluto, $\mathrm{kg}$ (Fresh weight, $\mathrm{kg}$ ) } \\
\hline $\begin{array}{l}\text { Rúmen-retículo** } \\
\text { Reticulum-rumen }\end{array}$ & $13,62 \pm 0,59 b$ & $15,82 \pm 0,59 a$ & $10,44 \pm 0,59 \mathrm{c}$ \\
\hline Abomaso* & $3,23 \pm 0,41 b$ & $3,72 \pm 0,41 \mathrm{a}$ & $2,57 \pm 0,41 b$ \\
\hline $\begin{array}{l}\text { Abomasum } \\
\text { Omaso** } \\
\text { Omasum }\end{array}$ & $5,17 \pm 0,64 b$ & $7,65 \pm 0,64 a$ & $7,84 \pm 0,64 a$ \\
\hline $\begin{array}{l}\text { Estômago** } \\
\text { Stomach }\end{array}$ & $22,03 \pm 1,03 b$ & $27,20 \pm 1,03 \mathrm{a}$ & $20,85 \pm 1,03 b$ \\
\hline Intestinos* & $16,51 \pm 1,50 \mathrm{a}$ & $21,19 \pm 1,50 \mathrm{a}$ & $21,24 \pm 1,5 \mathrm{a}$ \\
\hline $\begin{array}{l}\text { Intestines } \\
\text { TGI** } \\
\text { GIT }\end{array}$ & $38,53 \pm 1,85 b$ & $48,38 \pm 1,85 a$ & $42,09 \pm 1,85 b$ \\
\hline
\end{tabular}

$\%$ do peso de abate (\% of slaughter weight)

\begin{tabular}{|c|c|c|c|}
\hline Rúmen-retículo** & $2,94 \pm 0,11 \mathrm{a}$ & $3,10 \pm 0,11 \mathrm{a}$ & $1,85 \pm 0,11 \mathrm{~b}$ \\
\hline $\begin{array}{l}\text { Reticulum-rumen } \\
\text { Abomaso** }\end{array}$ & $0,70 \pm 0,08 \mathrm{a}$ & $074+0.08 \mathrm{a}$ & $0,45 \pm 0.08 b$ \\
\hline Abomasum & & & \\
\hline Omaso** & $1,08 \pm 0,13 b$ & $1,52 \pm 0,13 \mathrm{a}$ & $1,39 \pm 0,13 \mathrm{ab}$ \\
\hline $\begin{array}{l}\text { Omasum } \\
\text { Estômago** } \\
\text { Stomach }\end{array}$ & $4,73 \pm 0,20 b$ & $5,36 \pm 0,20 \mathrm{a}$ & $3,69 \pm 0,20 \mathrm{c}$ \\
\hline Intestinos & $3,59 \pm 0,23$ & $4,17 \pm 0,23$ & $3,71 \pm 0,23$ \\
\hline $\begin{array}{l}\text { Intestines } \\
\text { TGI** }\end{array}$ & $8,32 \pm 0,27 b$ & $9,53 \pm 0,27 \mathrm{a}$ & $7,41 \pm 0,27 \mathrm{c}$ \\
\hline GIT & & & \\
\hline & \multicolumn{3}{|c|}{$\%$ do corpo vazio (\% of empty body) } \\
\hline $\begin{array}{l}\text { Rúmen-retículo** } \\
\text { Reticulum-rumen }\end{array}$ & $3,62 \pm 0,12 \mathrm{a}$ & $3,81 \pm 0,12 \mathrm{a}$ & $2,26 \pm 0,12 b$ \\
\hline Abomaso* & $0,87 \pm 0,10 \mathrm{a}$ & $0,91 \pm 0,10 \mathrm{a}$ & $0,55 \pm 0,10 \mathrm{~b}$ \\
\hline $\begin{array}{l}\text { Abomasum } \\
\text { Omaso** }\end{array}$ & $1,34 \pm 0,17 b$ & $1,88 \pm 0,17 \mathrm{a}$ & $1,70 \pm 0,17 \mathrm{ab}$ \\
\hline $\begin{array}{l}\text { Omasum } \\
\text { Estômago** } \\
\text { Stomach }\end{array}$ & $5,83 \pm 0,26 b$ & $6,60 \pm 0,26 \mathrm{a}$ & $4,51 \pm 0,26 \mathrm{c}$ \\
\hline $\begin{array}{l}\text { Intestinos* } \\
\text { Intestines }\end{array}$ & $4,41 \pm 0,27 b$ & $5,14 \pm 0,27 \mathrm{a}$ & $4,53 \pm 0,27 \mathrm{ab}$ \\
\hline $\begin{array}{l}\text { TGI** } \\
\text { GIT }\end{array}$ & $10,24 \pm 0,34 b$ & $11,74 \pm 0,34 \mathrm{a}$ & $9,04 \pm 0,34 \mathrm{c}$ \\
\hline
\end{tabular}

$a, b, c$ : médias na mesma linha diferem ** $(P<0,05)$ ou * $(P<0,10)$ pelo teste t. $a, b, c$ : means in the same row $\operatorname{differ}^{* *}(P<0.05)$ or $(P<0.10)$ by $t$ test.

Angus ao tamanho de pulmão. Jorge \& Fontes (2001) descreveram incremento linear desses órgãos com o aumento da maturidade de novilhos de corte e relataram que o baço apresentou intensidade de crescimento superior ao PCV. Restle et al. (2005), por sua vez, registraram aumento linear 


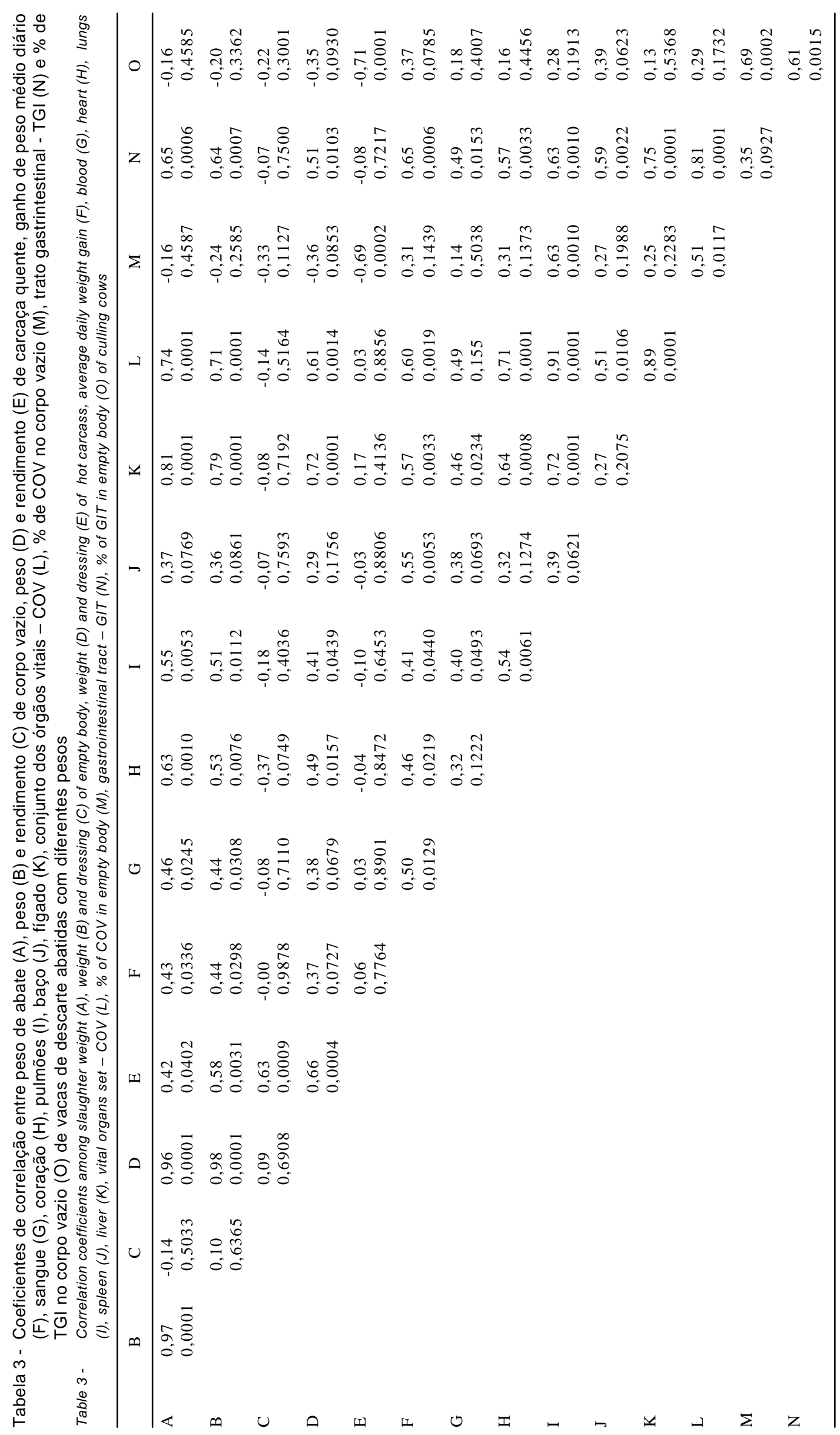


no peso dos pulmões e estagnação do baço frente ao aumento do PA e PCV em novilhos.

$\mathrm{O}$ peso do conjunto dos órgãos vitais (COV) em valores absolutos foi maior nos animais do T507 e T566, o que pode estar associado à maior quantidade de sangue nestes grupos de abate. A associação entre o desenvolvimento do COV e o PA foi de 0,74 (Tabela 3). Ribeiro et al. (2001) associaram o maior tamanho dos órgãos internos ao aumento proporcional de sangue no corpo de bezerros alimentados com diferentes proporções de concentrado na dieta.

Os valores relativos de COV para PA e PCV obtidos nos animais do T566 foram inferiores aos registrados dos de mais tratamentos. Segundo Galvão et al. (1991), os órgãos vitais têm maior desenvolvimento na fase mais precoce da vida do animal, ocasião em que sua taxa de desenvolvimento suplanta os crescimentos muscular e adiposo, situação contrária à medida que o animal cresce. Desse modo, os órgãos passam a representar menor proporção sobre o peso corporal vazio. Signoretti et al. (1999), em estudo com animais abatidos aos 190 e $300 \mathrm{~kg}$ de PV, também reportaram menor participação do $\mathrm{COV}$ no $\mathrm{PCV}$ nos animais mais pesados (4,65 e 4,06\%, respectivamente).

Apesar da ausência de diferença significativa para os resultados percentuais do PA e PCV para rúmen-retículo e abomaso entre T465 e T507, o T507 apresentou valores superiores para rúmen-retículo, abomaso, estômago e trato gastrintestinal em todas as formas de expressão (Tabela 2), o que está associado ao ganho compensatório destes

Tabela 4 - Pesos de corpo vazio, coração, fígado, pulmões, rins, baço e conjunto dos órgãos vitais (coração + fígado + pulmões + rins + baço) (COV) de vacas de descarte segundo a geração de cruzamento (G2 e G3) e o grupo genético (GG)

Table 4 - Means and standard errors for weights of empty body, heart, liver, lungs, kidneys, spleen, vital organs set (heart + liver + lungs + kidneys + spleen) (COV) according to crossbreeding generation (G2 and G3) and genetic group (GG)

\begin{tabular}{|c|c|c|c|c|c|c|c|c|}
\hline GG & $\begin{array}{l}\text { Corpo vazio } \\
\text { Empty body }\end{array}$ & $\begin{array}{l}\text { Sangue } \\
\text { Blood }\end{array}$ & $\begin{array}{c}\text { Coração } \\
\text { Heart }\end{array}$ & $\begin{array}{l}\text { Fígado } \\
\text { Liver }\end{array}$ & $\begin{array}{l}\text { Pulmões } \\
\text { Lungs }\end{array}$ & $\begin{array}{l}\text { Rins } \\
\text { Kidneys }\end{array}$ & $\begin{array}{l}\text { Baço } \\
\text { Spleen }\end{array}$ & $\mathrm{COV}$ \\
\hline & \multicolumn{8}{|c|}{ Peso absoluto, kg (Fresh weight, $\mathrm{kg}$ ) } \\
\hline $3 / 4 \mathrm{CN}$ & $417,45 \pm 13,17$ & $14,95 \pm 0,78 \mathrm{a}$ & $1,86 \pm 0,12 \mathrm{c}$ & $6,19 \pm 0,28$ & $5,70 \pm 0,30$ & $1,13 \pm 0,12 \mathrm{a}$ & $1,14 \pm 0,13$ & $16,01 \pm 0,54 \mathrm{a}$ \\
\hline $3 / 4 \mathrm{NC}$ & $404,90 \pm 14,07$ & $12,07 \pm 0,83 b$ & $1,50 \pm 0,13 \mathrm{~d}$ & $5,52 \pm 0,30$ & $4,94 \pm 0,30$ & $0,75 \pm 0,13 b$ & $0,95 \pm 0,14$ & $13,66 \pm 0,57 b$ \\
\hline $\mathrm{G} 2$ & $411,18 \pm 9,24$ & $13,51 \pm 0,55 \mathrm{~B}$ & $1,68 \pm 0,08$ & $5,85 \pm 0,19$ & $5,32 \pm 0,21$ & $0,94 \pm 0,08$ & $1,05 \pm 0,09$ & $14,84 \pm 0,38$ \\
\hline $5 / 8 \mathrm{CN}$ & $421,01 \pm 12,96$ & $16,16 \pm 0,77 \mathrm{a}$ & $1,71 \pm 0,12$ & $6,31 \pm 0,27$ & $5,72 \pm 0,30 \mathrm{c}$ & $0,79 \pm 0,12$ & $1,25 \pm 0,13$ & $15,77 \pm 0,53$ \\
\hline $5 / 8 \mathrm{NC}$ & $426,65 \pm 13,00$ & $14,23 \pm 0,77 b$ & $1,79 \pm 0,12$ & $6,22 \pm 0,27$ & $4,92 \pm 0,30 \mathrm{~d}$ & $0,73 \pm 0,12$ & $1,27 \pm 0,13$ & $14,94 \pm 0,53$ \\
\hline G3 & $423,83 \pm 9,24$ & $15,20 \pm 0,55 a$ & $1,75 \pm 0,08$ & $6,26 \pm 0,19$ & $5,32 \pm 0,21$ & $0,76 \pm 0,08$ & $1,26 \pm 0,09$ & $15,36 \pm 0,38$ \\
\hline
\end{tabular}

Diferença $\mathrm{a}^{1}$

$1,75 \pm 0,08 \quad 6,26 \pm 0,19 \quad 5,32 \pm 0,21$

$0,76 \pm 0,08$

$1,26 \pm 0,09$

$5,36 \pm 0,38$

Difference

$\mathrm{PC}-\mathrm{PN}$

3,45

$2,40^{*}$

0,14

0,38

$0,78 *$

$0,22 * *$

0,08

$1,59 *$

$\%$ do peso de abate (\% of slaughter weight)

$\begin{array}{lrrrrrrrr}3 / 4 \mathrm{CN} & - & 2,90 \pm 0,14 \mathrm{c} & 0,36 \pm 0,02 & 1,20 \pm 0,05 & 1,10 \pm 0,06 & 0,22 \pm 0,02 \mathrm{c} & 0,22 \pm 0,03 & 3,11 \pm 0,08 \mathrm{a} \\ 3 / 4 \mathrm{NC} & - & 2,51 \pm 0,15 \mathrm{~d} & 0,31 \pm 0,02 & 1,13 \pm 0,05 & 1,02 \pm 0,06 & 0,15 \pm 0,02 \mathrm{~d} & 0,19 \pm 0,03 & 2,81 \pm 0,08 \mathrm{~b} \\ \mathrm{G} 2 & - & 2,71 \pm 0,10 & 0,33 \pm 0,01 & 1,17 \pm 0,03 & 1,06 \pm 0,04 & 0,19 \pm 0,01 & 0,21 \pm 0,02 & 2,96 \pm 0,06 \\ 5 / 8 \mathrm{CN} & - & 3,14 \pm 0,14 \mathrm{c} & 0,33 \pm 0,02 & 1,22 \pm 0,05 & 1,10 \pm 0,06 \mathrm{c} & 0,16 \pm 0,02 & 0,24 \pm 0,02 & 3,05 \pm 0,08 \\ 5 / 8 \mathrm{NC} & - & 2,74 \pm 0,14 \mathrm{~d} & 0,34 \pm 0,02 & 1,19 \pm 0,05 & 0,94 \pm 0,06 \mathrm{~d} & 0,14 \pm 0,02 & 0,24 \pm 0,02 & 2,86 \pm 0,08 \\ \text { G3 } & - & 2,94 \pm 0,10 & 0,33 \pm 0,01 & 1,20 \pm 0,03 & 1,02 \pm 0,04 & 0,15 \pm 0,01 & 0,24 \pm 0,02 & 2,95 \pm 0,06\end{array}$

Diferença ${ }^{1}$

Difference

PC - PN

0,39

0,02

0,05

$0,12 *$

0,04

0,01

$0,24 *$

$\%$ do corpo vazio (\% of empty body)

$3 / 4 \mathrm{CN}$

$3 / 4 \mathrm{NC}$

G2

$5 / 8 \mathrm{CN}$

$5 / 8 \mathrm{NC}$

G3

Diferença $\mathrm{a}^{1}$

Difference

$\mathrm{PC}-\mathrm{PN}$

\begin{abstract}
$3,58 \pm 0,17 \mathrm{a}$
$3,06 \pm 0,18 \mathrm{~b}$

$3,32 \pm 0,12 \mathrm{C}$

$3,87 \pm 0,17 \mathrm{a}$

$3,36 \pm 0,17 \mathrm{~b}$

$3,62 \pm 0,12 \mathrm{D}$
\end{abstract}

$0,51 * *$
$0,45 \pm 0,03$

$0,38 \pm 0,03$

$0,41 \pm 0,02$

$0,41 \pm 0,03$

$0,41 \pm 0,03$

$0,41 \pm 0,02$
$1,36 \pm 0,07$

$1,24 \pm 0,07$

$1,30 \pm 0,05$

$1,36 \pm 0,07 \mathrm{a}$

$1,15 \pm 0,07 \mathrm{~b}$

$1,26 \pm 0,05$
$0,27 \pm 0,02 \mathrm{a}$

$0,19 \pm 0,03 \mathrm{~b}$

$0,23 \pm 0,02 \mathrm{~A}$

$0,19 \pm 0,03$

$0,18 \pm 0,02$

$0,18 \pm 0,02 \mathrm{~B}$

\section{$0,27 \pm 0,03$}

$0,23 \pm 0,03$

$0,25 \pm 0,02$

$0,30 \pm 003$

$0,30 \pm 0,03$

$0,30 \pm 0,03$
$3,84 \pm 0,10 \mathrm{a}$

$3,42 \pm 0,11 \mathrm{~b}$

$3,63 \pm 0,07$

$3,76 \pm 0,10 \mathrm{c}$

$3,50 \pm 0,10 \mathrm{~d}$

$3,63 \pm 0,07$

$a, b$ e $A, B$ : na mesma coluna diferem $(P<0,05)$ pelo teste t entre grupo genético e geração de cruzamento, respectivamente

c, d e $C$, D: na mesma coluna diferem $(P<0,10)$ pelo teste t entre grupo genético e geração de cruzamento, respectivamente.

${ }^{1}$ Contraste entre predominância $P C(3 / 4 C N+5 / 8 C N)$ e $P N(3 / 4 N C+5 / 8 N C)$.

** $(\mathrm{P}<0,05) ;{ }^{*}(\mathrm{P}<0,10)$.

${ }^{1}$ Contrast between $P C(3 / 4 C N+5 / 8 C N)$ and $P N(3 / 4 N C+5 / 8 N C)$ predominance.

$a, b$ and $A, B$ in the same column differ $(P<0.05)$ by $t$ test between genetic group and crossbreeding generation, respectively.

$c, d$ and $C, D$ in same column differ $(P<0.10)$ by t test between genetic group and crossbreeding generation, respectively.

${ }^{\star *}(P<0.05) ;{ }^{*}(P<0.10)$ 
animais até este peso de abate, visto que as vacas se encontravam magras ao início do experimento (condição corporal média de 2,35 pontos). Segundo Jorge et al. (1999), animais sob restrição alimentar apresentam redução do volume destes compartimentos e posterior recuperação mediante realimentação à vontade (Hoog, 1991).

A correlação entre o desenvolvimento do trato gastrintestinal e o ganho de peso foi de 0,65 (Tabela 3 ) e pode ser atribuída às maiores taxas de ganho de peso resultantes do maior consumo de alimento. Entretanto, quando os animais foram abatidos aos $566 \mathrm{~kg}$, foram observados menores valores para o estômago, ocasionados, em parte, pela pressão interna realizada pela gordura presente na cavidade abdominal com o aumento do peso de 507 para $566 \mathrm{~kg}$ (Kuss et al., 2007).

Conforme descrito pelo NRC (1996), à medida que avança o grau de maturidade, observa-se diminuição do consumo por unidade de peso metabólico, relacionada à redução de apetite e, principalmente, à competição de espaço abdominal e ao limite do potencial de deposição de gordura.

Como descrito na Tabela 4, as médias absolutas para peso de coração, rins e COV obtidas nos animais 3/4CN foram superiores às obtidas com as vacas 3/4NC. Quando expressos em valores percentuais do PA e PCV, apenas os pesos de rins e COV diferiram significativamente. Animais $5 / 8 \mathrm{CN}$ foram superiores aos $5 / 8 \mathrm{NC}$ quanto aos pesos de

Tabela 5 - Pesos de rúmen-retículo, abomaso, omaso, estomago (rúmen-retículo + abomaso + omaso), intestinos (delgado + grosso), trato gastrintestinal (estômago + intestinos) (TGI), TGI/100 kg de corpo vazio (CV) de vacas de descarte segundo a geração de cruzamento (G2 e G3) e o grupo genético (GG)

Table 5 - Means and standard errors for weights of reticulum-rumen, abomasum, omasum, stomach (reticulum-rumen + abomasum + omasum), intestines (small + large), gastrointestinal tract (stomach + intestines) (GIT), GIT/100 kg of empty body (EB) according to crossbreeding generation (G2 and G3) and genetic group (GG)

\begin{tabular}{|c|c|c|c|c|c|c|}
\hline GG & $\begin{array}{l}\text { Rúmen-retículo } \\
\text { Reticulum-rumen }\end{array}$ & $\begin{array}{l}\text { Abomaso } \\
\text { Abomasum }\end{array}$ & $\begin{array}{l}\text { Omaso } \\
\text { Omasum }\end{array}$ & $\begin{array}{c}\text { Estômago } \\
\text { Stomach }\end{array}$ & $\begin{array}{l}\text { Intestinos } \\
\text { Intestines }\end{array}$ & $\begin{array}{l}\text { TGI } \\
G I T\end{array}$ \\
\hline & \multicolumn{6}{|c|}{ Peso absoluto, kg (Fresh weight, kg) } \\
\hline $3 / 4 \mathrm{CN}$ & $12,83 \pm 0,70$ & $3,47 \pm 0,49$ & $6,58 \pm 0,75$ & $22,89 \pm 1,21$ & $19,85 \pm 1,77$ & $42,73 \pm 2,18$ \\
\hline $3 / 4 \mathrm{NC}$ & $11,77 \pm 0,75$ & $3,39 \pm 0,52$ & $6,52 \pm 0,80$ & $21,68 \pm 1,29$ & $18,10 \pm 1,89$ & $39,78 \pm 2,33$ \\
\hline $\mathrm{G} 2$ & $12,30 \pm 0,49 \mathrm{~B}$ & $3,43 \pm 0,34$ & $6,55 \pm 0,52$ & $22,28 \pm 0,85 \mathrm{D}$ & $18,97 \pm 1,24$ & $41,26 \pm 1,53$ \\
\hline $5 / 8 \mathrm{CN}$ & $14,48 \pm 0,69$ & $3,07 \pm 0,48$ & $7,38 \pm 0,74$ & $24,92 \pm 1,19$ & $20,04 \pm 1,74$ & $44,96 \pm 2,14$ \\
\hline $5 / 8 \mathrm{NC}$ & $14,11 \pm 0,69$ & $2,77 \pm 0,48$ & $7,06 \pm 0,74$ & $23,94 \pm 1,19$ & $20,59 \pm 1,75$ & $44,54 \pm 2,15$ \\
\hline G3 & $14,29 \pm 0,49 \mathrm{~A}$ & $2,92 \pm 0,34$ & $7,22 \pm 0,52$ & $24,43 \pm 0,85 \mathrm{C}$ & $20,32 \pm 1,24$ & $44,75 \pm 1,53$ \\
\hline \multicolumn{7}{|l|}{ Diferença ${ }^{1}$} \\
\hline \multicolumn{7}{|l|}{ Difference } \\
\hline \multirow[t]{2}{*}{$\mathrm{PC}-\mathrm{PN}$} & 0,71 & 0,19 & 0,19 & 1,10 & 0,60 & 1,68 \\
\hline & \multicolumn{6}{|c|}{$\%$ do peso de abate (\% of slaughter weight) } \\
\hline $3 / 4 \mathrm{CN}$ & $2,49 \pm 0,13$ & $0,67 \pm 0,10$ & $1,27 \pm 0,16$ & $4,43 \pm 0,23$ & $3,87 \pm 0,27$ & $8,30 \pm 0,32$ \\
\hline $3 / 4 \mathrm{NC}$ & $2,46 \pm 0,14$ & $0,71 \pm 0,10$ & $1,26 \pm 0,17$ & $4,43 \pm 0,25$ & $3,67 \pm 0,29$ & $8,10 \pm 0,34$ \\
\hline G2 & $2,48 \pm 0,09 \mathrm{~B}$ & $0,69 \pm 0,07$ & $1,27 \pm 0,11$ & $4,44 \pm 0,16$ & $3,77 \pm 0,19$ & $8,20 \pm 0,23$ \\
\hline $5 / 8 \mathrm{CN}$ & $2,85 \pm 0,13$ & $0,62 \pm 0,10$ & $1,43 \pm 0,16$ & $4,90 \pm 0,23$ & $3,88 \pm 0,26$ & $8,79 \pm 0,32$ \\
\hline $5 / 8 \mathrm{NC}$ & $2,72 \pm 0,13$ & $0,53 \pm 0,10$ & $1,35 \pm 0,16$ & $4,60 \pm 0,23$ & $3,88 \pm 0,26$ & $8,48 \pm 0,32$ \\
\hline G3 & $2,78 \pm 0,09 \mathrm{~A}$ & $0,57 \pm 0,07$ & $1,39 \pm 0,11$ & $4,75 \pm 0,16$ & $3,88 \pm 0,19$ & $8,63 \pm 0,23$ \\
\hline \multicolumn{7}{|l|}{ Diferença $^{1}$} \\
\hline \multirow{3}{*}{$\begin{array}{l}\text { Difference } \\
\mathrm{PC}-\mathrm{PN} \\
\end{array}$} & & & & & & \\
\hline & 0,08 & 0,02 & 0,05 & 0,15 & 0,09 & 0,25 \\
\hline & \multicolumn{6}{|c|}{$\%$ do corpo vazio (\% of empty body) } \\
\hline $3 / 4 \mathrm{CN}$ & $3,09 \pm 0,15$ & $0,84 \pm 0,12$ & $1,58 \pm 0,20$ & $5,51 \pm 0,31$ & $4,77 \pm 0,32$ & $10,28 \pm 0,40$ \\
\hline $3 / 4 \mathrm{NC}$ & $3,00 \pm 0,16$ & $0,86 \pm 0,13$ & $1,55 \pm 0,22$ & $5,40 \pm 0,33$ & $4,45 \pm 0,34$ & $9,85 \pm 0,43$ \\
\hline $\mathrm{G} 2$ & $3,04 \pm 0,10 \mathrm{~B}$ & $0,84 \pm 0,08$ & $1,57 \pm 0,14$ & $5,46 \pm 0,21$ & $4,61 \pm 0,23$ & $10,07 \pm 0,28$ \\
\hline $5 / 8 \mathrm{CN}$ & $3,51 \pm 0,14$ & $0,76 \pm 0,12$ & $1,77 \pm 0,20$ & $6,03 \pm 0,30$ & $4,80 \pm 0,32$ & $10,83 \pm 0,40$ \\
\hline $5 / 8 \mathrm{NC}$ & $3,32 \pm 0,14$ & $0,65 \pm 0,12$ & $1,66 \pm 0,20$ & $5,63 \pm 0,30$ & $4,76 \pm 0,32$ & $10,39 \pm 0,40$ \\
\hline G3 & $3,42 \pm 0,10 \mathrm{~A}$ & $0,70 \pm 0,08$ & $1,71 \pm 0,14$ & $5,83 \pm 0,21$ & $4,78 \pm 0,23$ & $10,07 \pm 0,28$ \\
\hline \multicolumn{7}{|l|}{ Diferença $^{1}$} \\
\hline \multicolumn{7}{|l|}{ Difference } \\
\hline $\mathrm{PC}-\mathrm{PN}$ & 0,14 & 0,06 & 0,07 & 0,25 & 0,18 & 0,43 \\
\hline
\end{tabular}

$A$, B: na mesma coluna diferem $(P<0,05)$ pelo teste $t$ entre geração.

$C$, D: na mesma coluna diferem $(P<0,10)$ pelo teste t entre geração.

1. Contraste entre Predominância $\mathrm{PC}(3 / 4 \mathrm{CN}+5 / 8 \mathrm{CN})$ e $\mathrm{PN}(3 / 4 \mathrm{NC}+5 / 8 \mathrm{NC})$

$A, B$ : in the same column differ $(P<0.05)$ by test $t$ between generation.

$C, D$ : in the same column differ $(P<0.10)$ by test $t$ between generation.

1: Contrast between $P C(3 / 4 C N+5 / 8 C N)$ and $P N(3 / 4 N C+5 / 8 N C)$ predominance. 
pulmão (kg e \% do PA e do $\mathrm{PCV}$ ) e à porcentagem de COV no $\mathrm{CV}$. Esse comportamento pode estar associado ao maior peso e, conseqüentemente, ao maior fluxo sanguíneo nas vacas de predominância Charolês em relação às de predominância Nelore $(15,55$ vs $13,15 \mathrm{~kg}$, respectivamente, Tabela 4), resultando em aumento no tamanho destes órgãos e, conseqüentemente, do conjunto dos órgãos internos (COV). Ribeiro et al. (2001) associaram o maior tamanho relativo dos órgãos à maior quantidade de sangue no corpo dos animais. Neste estudo, a quantidade desse fluido acompanhou o desenvolvimento do PA e do PCV dos animais, com exceção das vacas $5 / 8 \mathrm{NC}$, que apresentaram comportamento contrário. Os resultados comprovaram que, quanto maior a deposição de tecidos, maior o aporte de sangue exigido para condução de nutrientes. $\mathrm{O}$ aumento do peso vivo ao abate também resultou em incremento na quantidade de sangue em novilhos $5 / 8 \mathrm{NC}$, porém, sua participação no PCV se manteve estável (Restle et al., 2005). Em novilhos mestiços leiteiros e Zebu, Backes et al. (2003) não verificaramalteração na quantidade de sangue entre os grupos genéticos estudados.

Superioridade no tamanho de coração, pulmão, fígado e COV de novilhos Charolês em relação aos Nelores foi relatada por Menezes et al. (2007). Ferrel \& Jenkins (1998) reportaram maiores valores de coração, fígado, pulmão, rins e baço para animais Angus e Hereford em relação ao Brahman. Lunt et al. (1986) observaram que o tamanho de coração, fígado e pulmões nos novilhos Angus foi maior que nos animais Brahman e Angus x Brahman. Pacheco et al. (2005) verificaram maior desenvolvimento do fígado $(\mathrm{kg}, \%$ do PA e do PCV) em novilhos 5/8CN em relação aos 5/8 NC terminados nos sistemas jovem (22-24 meses) e superjovem (12-14 meses).

Estudos indicam que o desenvolvimento do COV está relacionado ao maior consumo de energia da dieta (Signoretti et al., 1999; Ferreira et al., 2000; Ribeiro et al., 2001) ou ao consumo de alimento (Jorge et al., 1999). Animais destinados à produção de carne, de porte grande (como os Charolês), selecionados para elevadas taxas de ganho de peso, apresentam maior consumo de alimento, sendo necessário aumento simultâneo dos órgãos responsáveis pelo metabolismo basal. Segundo Jones et al. (1985), animais originados do cruzamento com raças de corte de porte grande apresentaram maiores pesos de coração, baço, fígado e pulmões em relação àqueles oriundos do cruzamento de raças pequenas. Ferrel et al. (1976) descreveram que as diferenças nos requerimentos nutricionais entre grupos genéticos podem ser atribuídas também aos diferentes tamanhos dos órgãos internos, que são maiores em animais taurinos em comparação aos zebuínos.
O desenvolvimento do rúmen-retículo e do estômago (em $\mathrm{kg}$ ) foi maior nas vacas G3 em comparação às G2 (Tabela 5), todavia, apenas o rúmen-retículo foi influenciado pelo PA e o PCV. Este fato está relacionado ao maior peso dos animais da G3 (521,5 vs 503,6 kg - Kuss et al., 2005), como resultado do maior grau de heterozigose.Menezes \& Restle (2005) verificaram que, em novilhos da G2 e da G3, a heterose para peso e consumo alimentar acompanhou o grau da heterozigose.

\section{Conclusões}

Entre os órgãos, o fígado apresentou maior ímpeto de desenvolvimento com o aumento do peso de abate de vacas de descarte.

A participação relativa dos órgãos vitais e do trato gastrintestinal no peso vivo ao abate e no peso de corpo vazio foi menor nas vacas mais pesadas.

Animais da terceira geração provenientes do cruzamento Charolês $\times$ Nelore apresentaram maior desenvolvimento do rúmen-retículo e do estômago.

Maior participação de Charolês no genótipo conferiu maior volume de sangue e desenvolvimento dos pulmões e do conjunto dos órgãos vitais no corpo vazio.

\section{Literatura Citada}

ANUALPEC 2004. Anuário da Pecuária Brasileira. São Paulo: FNP Consultoria \& Comercio, 2004. 376p.

BACKES, A.A.; PAULINO, M.; ALVES, D.D. et al. Tamanho relativo dos órgãos internos e do trato gastrintestinal de bovinos zebu e mestiços leiteiros: I. Recria. In: REUNIÃO ANUAL DA SOCIEDADE BRASILEIRA DE ZOOTECNIA, 40., 2003, Santa Maria. Anais... Santa Maria: Sociedade Brasileira de Zootecnia, 2003. (CD-ROM).

CATON, J.S.; DHUYVETTER, D.V. Influence of energy supplementation on grazing ruminants: requirements and responses. Journal of Animal Science, v.75, n.1, p.533$542,1997$.

FERNANDES, H.J.; PAULINO, M.F.; MARTINS, R.G.R. et al. Crescimento de components corporais de três grupos genéticos nas fase de recria e terminação. Revista Brasileira de Zootecnia, v.34, p.288-296, 2005.

FERREIRA, M.A.; VALADARES FILHO, S.C.; MUNIZ, E.B. et al. Características das carcaças, biometria do trato gastrintestinal, tamanho dos órgãos internos e conteúdo gastrintestinal de bovinos F1 Simental x Nelore alimentados com dietas contendo vários níveis de concentrado. Revista Brasileira de Zootecnia, v.29, n.4, p.1174-1182, 2000.

FERREL, C.L.; GARRET, W.N.; HINMAN, N. Estimation of body composition in pregnant and non pregnant heifers. Journal of Animal Science, v.42, n.5, p.1158-1166, 1976.

FERRELL, C.L.; JENKINS, T.G. Body composition and energy utilization by steers of diverse genotypes fed a high-concentrate diet during the finishing period: II. Angus, Boran, Brahman, Hereford, and Tuli sires. Journal of Animal Science, v.76, p.647-657, 1998 
GALVÃO, J.G.; FONTES, C.A.A.; PIRES, C.C. et al. Característica e composição física da carcaça de bovinos não-castrados, abatidos em diferentes estágios de maturidade, de três grupos raciais (Estudo II). Revista da Sociedade Brasileira de Zootecnia, v.20, n.5, p.502-512, 1991.

HOOG, B.W. Compensatory growth in ruminants. In: PERSON, A.M.; DUTSON, T.R. (Ed.). Growth regulation in farm animals. London: Elsevier Applied Science, 1991. p.103-134.

JONES, S.D.M.; ROMPALA, R.E.; JEREMIAH, L.E. Growth and composition of the empty body in steers of different maturity types fed concentrate or forage diets. Journal of Animal Science, v.60, n.2, p.427-433, 1985

JORGE, A.M.; FONTES, C.A.A. Desenvolvimento relativo das partes do corpo de zebuínos de quatro raças. Ciência Rural, v.31, n.5, p.857-861, 2001.

JORGE, A.M.; FONTES, C.A.A.; PAULINO, M.F. et al. Tamanho relativo dos órgãos internos de zebuínos sob alimentação restrita e ad libitum. Revista Brasileira de Zootecnia, v.28, n.2, p.374-380, 1999.

KUSS, F.; RESTLE, J.; BRONDANI, I.L. et al. Características da carcaça de vacas de descarte de diferentes grupos genéticos terminados em confinamento com distintos pesos. Revista Brasileira de Zootecnia, v.34, n.3, p.915-925, 2005 (supl.).

KUSS, F.; RESTLE, J.; BRONDANI, I.L. et al. Componentes externos do corpo e gordura de descarte em vacas mestiças Charolês - Nelore, abatidas com pesos distintos Revista Brasileira de Zootecnia, 2007 (no prelo).

LUNT, D.K.; BYERS, F.M.; GREENE, L.W. et al. Effects of breed, diet, and growth rate on vital organ mass in growing and finishing beef steers. Journal of Animal Science, v.63, n.1, p.70-71, 1986 (suppl. 1).

MENEZES, L.F.G.; RESTLE, J. Desempenho de novilhos de gerações avançadas do cruzamento alternado entre as raças Charolês e Nelore, terminados em confinamento. Revista Brasileira de Zootecnia, v.34, n.6, p.1927-1937, 2005

MENEZES, L.F.G.; RESTLE, J.; BRONDANI, I.L. et al. Órgãos internos e trato gastrintestinal de novilhos de gerações avançadas do cruzamento rotativo entre as raças Charolês e Nelore terminados em confinamento Revista Brasileira de Zootecnia, v.36, n.1, p.120-129, 2007.

NATIONAL RESEARCH COUNCIL - NRC. Nutrient requirements of beef cattle. 7.rev.ed. Washington, D.C.: National Academy Press, 1996. 242p.

OLIVEIRA, R.F.M.; FONTES, C.A.A.; CARNEIRO, L.H.D.M. et al. Biometria do trato gastrintestinal de bovinos de três grupos genéticos. Revista da Sociedade Brasileira de Zootecnia, v.21, n.2, p.205-210, 1992.

PACHECO, P.S.; RESTLE, J.; SILVA, J.H.S. et al. Características das partes do corpo não-integrantes da carcaça de novilhos jovens e superjovens de diferentes grupos genéticos. Revista Brasileira de Zootecnia, v.34, n.5, p.1678-1690, 2005.
RESTLE J. Comportamento reprodutivo do rebanho de gado de corte da fazenda experimental de criação experimental agronômica da UFRGS. $1^{\circ}$ Semestre. Seminário da disciplina de Técnicas de Pesquisas. Curso de Pós-Graduação em Agronomia, Universidade Federal do Rio Grande do Sul, 1972.

RESTLE, J.; MENEZES, L.F.G.; ARBOITTE, M.Z. et al. Características das partes não-integrante da carcaça de novilhos 5/6 Nelore 3/8 Charolês abatidos em três estádios de desenvolvimento. Revista Brasileira de Zootecnia, v.34, n.4, p.1339-1348, 2005.

RIBEIRO, T.R.; PEREIRA, J.C.; LEÃO, M.I. et al. Tamanho de órgãos e vísceras de bezerros holandeses, para produção de vitelos recebendo dietas com diferentes níveis de concentrado. Revista Brasileira de Zootecnia, v.30, n.6, p.2163-2168, 2001.

STATISTICAL ANALYSIS SYSTEM - SAS. SAS/STAT user's guide statistics. 4.ed. Cary: 1997. v.2, 943p.

SIGNORETTI, R.D.; ARAÚJO, G.G.L.; SILVA, J.F.C. et al. Características quantitativas da partes do corpo não-integrante da carcaça animal e desenvolvimento do trato gastrintestinal e bezerros da raça holandesa alimentados com dietas contendo quatro níveis de concentrado. Revista Brasileira de Zootecnia, v.28, n.4, p.875-882, 1999.

SMITH, N.E.; BALDWIN, R.L. Effects of breed, pregnancy, and lactation on weight of organs and tissues in dairy cattle. Journal of Dairy Science, v.57, n.9, p.1055-1060, 1973.

SOLIS, J.C.; BYERS, F.M.; SCHELLING, G.T. et al. Maintenance requirements and energetic efficiency of cows of different breed types. Journal of Animal Science, v.66, p.764-773, 1988.

VAZ, F.N.; RESTLE, J.; ALVES FILHO, D.C. et al. Peso das vísceras e rendimento de carcaças de novilhos ou novilhas Braford superprecoce, terminados com suplementação em pastagem cultivada sob pastejo controlado. In: REUNIÃO ANUAL DA SOCIEDADE BRASILEIRA DE ZOOTECNIA, 38., 2001, Piracicaba. Anais... Piracicaba: Sociedade Brasileira de Zootecnia, 2001. (CD-ROM).

VÉRAS, A.S.C.; VALADADRES FILHO, S.C.; SILVA, J.F.C. et al. Efeito do nível de concentrado sobre o peso dos órgãos internos e do conteúdo gastrintestinal de bovinos Nelore não-castrados. Revista Brasileira de Zootecnia, v.30, n.3, p.1120-1126, 2001 (supl.). 